\title{
Experiência Clínica com o Uso Conjunto de Sibutramina e Orlistat em Pacientes Obesos
}

\section{RESUMO}

Avaliamos a eficácia e a tolerabilidade da associação de sibutramina (10mg) e orlistat (120mg três vezes por dia às refeiçōes) no tratamento da obesidade em um estudo aberto de três meses de duração. $O$ estudo envolveu 114 pacientes não diabéticos com sobrepeso ou obesidade em busca de tratamento em clínica privada. Após dois meses, a perda média de peso foi $7,1 \mathrm{~kg}(-6,5 \%)$ e após três meses, a perda média de peso foi $8,9 \mathrm{~kg}(-8,2 \%)$. A associação de sibutramina e orlistat parece ser uma opção útil de tratamento da obesidade. (Arq Bras Endocrinol Metab 2000; 44/1: 103-5)

Unitermos: Sibutramina; Orlistat; Obesidade; Tratamento da obesidade.

\section{artigo original}

\author{
Alfredo Halpern \\ Ana Paola Monegaglia \\ Anna Beatriz G. Oliva \\ Monica Beyruti \\ Zuleika S.C. Halpern \\ Marcio C. Mancini
}

\begin{abstract}
The efficacy and tolerability of the association of sibutramine (10mg) and orlistat (120mg t.i.d.) in the treatment of obesity were evaluated in a 3 month short-term open study. The study involved 114 overweight or obese non-diabetic patients searching for obesity treatment in a private clinic. After two months, the mean weight loss was $7.1 \mathrm{~kg}(-6.5 \%)$ and after three months, the mean weight loss was $8.9 \mathrm{~kg}(-8.2 \%)$. The association of sibutramine and orlistat seems to be a useful option for the treatment of obesity. (Arq Bras Endocrinol Metab 2000; 44/1: 103-5)
\end{abstract}

Keywords: Sibutramine; Orlistat; Obesity: Treatment of obesity.
Besidade É UMA DOENÇA CRÓNiCA de prevalência crescente (l). Obesidade induz anormalidades metabólicas que contribuem para o desenvolvimento de diabetes mellitus e doenças cardiovasculares que requerem tratamento a longo prazo e estão associadas a risco elevado de morbidade e mortalidade $(2,3)$.

Dieta, modificações comportamentais e exercícios com freqüência falham em promover perda de peso consistente e mantida (4,5). O tratamento farmacológico pode aumentar a motivação do paciente e a aderência ao tratamento, sendo algumas vezes considerado uma estratégia necessária.

Entre os medicamentos contra a obesidade, a sibutramina (Knoll Pharmaceutical Company) e seus metabólitos agem no sistema nervoso central, inibindo a recaptação da serotonina e noradrenalina sem atividade de liberação e, dessa forma, modulando a neurotransmissão e aumentando a saciação e a saciedade $(6,7)$. Estudos em animais tratados cronicamente sugerem que a sibutramina tem propriedades termogênicas, afetando o balanço energético por aumentar a taxa metabólica via receptor adrenérgi$\cos \beta_{3}(6,8)$.

Orlistat (Hoffman-La Roche), um derivado hidrogenado da lipstatina sintetizado quimicamente, age por inibição parcial da atividade das lipases gástrica, pancreática e carboxil-ester no trato gastro-intestinal, reduzindo a
Clinica Alfredo Halpern São Paulo, SP. 
hidrólise dos triglicérides ingeridos e impedindo a absorção de aproximadamente $30 \%$ da gordura dietária ingerida $(9,10)$.

Uma vez que o mecanismo de ação desses dois fármacos é totalmente diverso, em teoria, é possível c razoável associá-los, obtendo desta forma benefício de ambos os modos de ação.

Utilizamos a combinação de sibutramina na dosagem de $10 \mathrm{mg}$ uma vez por dia pela manhã c orlistat $120 \mathrm{mg}$ três vezes por dia às refeições principais, por até três meses, em pacientes que buscaram tratamento para obesidade com o objetivo de analisar a eficiência e a tolerabilidade dessa associação a curto prazo.

\section{PACIENTES E MÉTODOS}

Foram analisados os resultados do tratamento com sibutramina $10 \mathrm{mg}$ uma vez por dia c orlistat $120 \mathrm{mg}$ três vezes por dia associada a uma dieta hipocalórica e recomendações de atividade fisica regular, em 114 pacientes com sobrepeso ou obesidade ( 52 homens e 62 mulheres), que retornaram pelo menos uma vez à consulta. Todos os pacientes eram de clínica privada. Os resultado estão mostrados na Tabela 1 . O paciente de 15 anos de idade sofria de obesidade grave (IMC = $32,5 \mathrm{~kg} / \mathrm{m}^{2}$ ) e os pacientes com IMC $<30 \mathrm{~kg} / \mathrm{m}^{2}$ tinham pelo menos duas comorbidades associadas à obesidade (hipertensão arterial sistêmica, dislipidemia ou problemas ortopédicos).

Todos os pacientes receberam prescrição de uma dieta hipocalórica, contendo atế $30 \%$ do conteúdo energético sob a forma de gordura, por nutricionista experimentada. A prescrição da dieta baseou-se em entrevista individual. Na primeira visita, técnicas de mudanças comportamentais foram orientadas e atividade física foi também encorajada. Os pacientes foram avaliados $\mathrm{em}$ visitas periódicas a cada duas semanas durante um período de dois ( 72 pacientes) e três (61 pacientes) meses. Peso, freqüência cardíaca, pressão artcrial e efeitos adversos foram registrados. O potencial relacionamento do efeito adverso apresentado com o tratamento foi julgado pelo investigador. Termos padronizados foram desenvolvidos para descrever eventos gastrintestinais possíveis devido ao aumento da excreção de gordura devido ao tratamento com orlistat.

Tabela 1. Características dos pacientes que entraram no estudo.

\begin{tabular}{lc}
\hline$N$, sexo & $114(62$ mulheres, 52 homens $)$ \\
IMC $\left(\mathrm{kg} / \mathrm{m}^{2}\right)$ & $37,3(27,8-54,3)$ \\
Idade (anos) & $43(15-72)$ \\
Peso $(\mathrm{Kg})$ & $108,6(65,7-183)$ \\
\hline
\end{tabular}

\section{RESULTADOS}

\section{Seguimento e avaliação da perda ponderal}

Setenta e dois dos 114 pacientes que iniciaram o estudo foram avaliados nos primeiros dois meses de seguimento. Quarenta e dois pacientes foram excluídos do estudo por má aderência (36) ou colaterais (6). Após dois meses, a diminuição média do peso corporal em relação aos valores iniciais foi $7,1 \mathrm{~kg}(6,5 \%)$. Sessenta e dois, 48 e 24 pacientes, respectivamente, perderam mais que $3 \%, 5 \%$ e $10 \%$ do peso corporal inicial.

Outros 11 pacientes foram excluídos no terceiro mês, todos por má aderência, de modo que 61 pacientes puderam ser avaliados nesse período. Após três meses, a redução média do peso corporal foi $8,9 \mathrm{~kg}(8,2 \%)$. Cinqüenta e três, 49 e 27 pacientes, respectivamente, perderam mais que $3 \%, 5 \%$ e $10 \%$ do peso inicial.

\section{Efeitos colaterais}

Oitenta e nove pacientes relataram pelo menos um efeito adverso, enquanto que 25 pacientes não apresentaram nenhum efeito colateral. Alguns eventos gastrintestinais foram atribuídos ao orlistat, enquanto outros eventos, no julgamento do investigador princi-

Tabela 2. Efeitos adversos remotamente, possivelmente ou provavelmente relacionados ao tratamento com orlistat.

\begin{tabular}{lcc}
\hline Efeito adverso & $N$ & $\%$ \\
& & \\
Fezes oleosas & $63 / 114$ & 55,2 \\
Aumento da evacuação & $16 / 114$ & 14,0 \\
Evacuação oleosa & $14 / 114$ & 12,2 \\
Flatus com descarga oleosa & $13 / 114$ & 11,4 \\
Urgência fecal & $4 / 114$ & 3,5 \\
Incontinência fecal & $5 / 114$ & 4,3 \\
Flatulência & $5 / 114$ & 4,3 \\
Diminuição da consistência & $9 / 114$ & 7,8 \\
\hline
\end{tabular}

Tabela 3. Efeitos adversos remotamente, possivelmente ou provavelmente relacionados ao tratamento com sibutramina

\begin{tabular}{lcc}
\hline Efeito adverso & $\mathrm{N}$ & $\%$ \\
Aumento de apetite & $9 / 114$ & 7.8 \\
Boca seca & $12 / 114$ & 10,5 \\
Obstipação & $3 / 114$ & 2,6 \\
Insônia & $4 / 114$ & 3.5 \\
Ansiedade & $2 / 114$ & 1.7 \\
Sudorese & $2 / 114$ & 1.7 \\
Irritabilidade & $2 / 174$ & 1.7 \\
Cefaléla & $2 / 114$ & 1.7 \\
Tontura & $2 / 114$ & 1.7 \\
Elevação da pressão & $2 / 114$ & 1.7 \\
Anorexia & $3 / 114$ & 2.6 \\
\hline
\end{tabular}

Analisando os pacientes que completaram o estudo em 3 meses de tratamento 
Tabela 4. Variação antropométrica nos pacientes que completaram o estudo.

Três meses

$N$

Sexo

IMC inicial

IMC final

Variação do IMC

Peso inicial

Peso final

Variação do peso

61

33 mulheres, 28 homens

$37.1 \mathrm{~kg} / \mathrm{m}^{2}$

$34,0 \mathrm{~kg} / \mathrm{m}^{2}$

$-3,1 \mathrm{~kg} / \mathrm{m}^{2}(-8,3 \%)$

$108,9 \mathrm{~kg}$

$99.7 \mathrm{~kg}$

$-9,2 \mathrm{~kg}(-8,4 \%)$

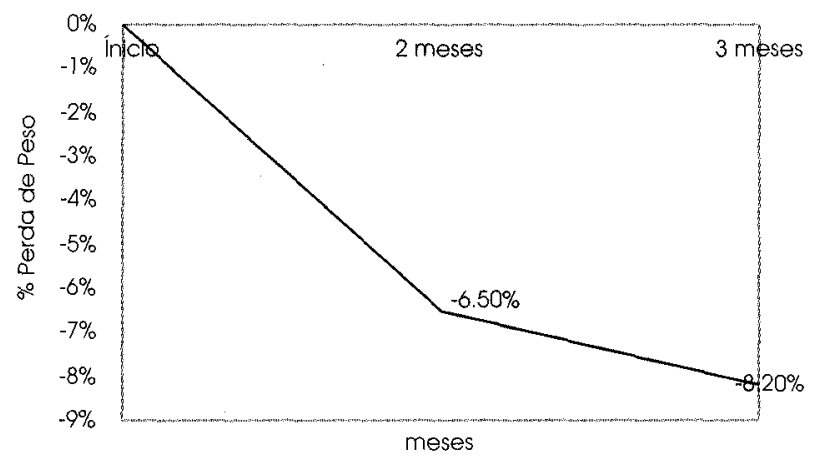

Gráfico 1. \% Perda ponderal após 2 e 3 meses de tratamento com associação de Sibutramina e Orlistat (Intention to Treat).

pal $(\mathrm{AH})$, estiveram relacionados com o tratamento com sibutramina (Tabelas 2 e 3). Não foi observada elevação significativa na freqüência cardíaca (estabelecida como uma variação maior que cinco batimentos por minuto) ou na pressão arterial (estabelecida como uma variação maior que $4 \mathrm{mmHg}$ ).

\section{DISCUSSÃO}

O tratamento farmacológico da obesidade é indicado em caso de falha do tratamento convencional (orientação dietética e exercício físico).

Sibutramina e orlistat são novos medicamentos com mecanismos de ação distintos.

O presente estudo é a primeira documentação de uma experiência clínica com a combinação de sibutramina e orlistat no tratamento da obesidade. A perda de peso foi evidente e o número (e a porcentagem) de indivíduos que perderam pelo menos $5 \%$ do peso corpóreo inicial nos primeiros dois meses com a associação de sibutramina e orlistat (75\%) foi comparável ao número obtido com sibutramina e orlistat em monoterapia em estudos de seis meses de duração (11-14).
A maioria dos efeitos adversos relatados neste estudos foram compatíveis com a farmacologia conhecida de sibutramina e orlistat.

Em conclusão, a combinação de sibutramina c orlistat é bem tolerada e eficaz em aumentar a perda de peso em pacientes obesos por um período de até três meses.

\section{REFERÊNCIAS}

1. Seidell JC. Obesity in Europe - scaling an epidemic. Int J Obes 1995: 19(suppl.3):S1-S4.

2. Pi-Sunyer PX. Medical hazards of obesity. Ann Intern Med 1993: 19:655-60.

3. Bray GA. Health hazards of obesity. Endocrinol Metab Clin N Am 1996:25:907-19.

4. Thomas PR (ed.). Weighing the Options: Criteria for Evaluating Weight-Management Programs. National Academy Press: Washington D.C. 1996.

5. National Task Force on the Prevention and Treatment of Obesity. Long-term pharmacotherapy in the management of obesity. JAMA 1996;276:1907-15.

6. Connoley IP, Heal DJ, Stock MJ. A study in rats of the effects of sibutramine on food intake and thermogenesis. Br J Pharmacol 1995: 1 14(suppl.):388P.

7. Halford JCG, Heal DJ, Blundell JE. Effects in the rat of sibutramine on food intake and the behavioral satiety sequence. Br J Pharmacol 1995; 1 14(suppl.):387P.

8. Connoley IP, Liu Y-L, Frost I, et al. Thermogenic effects of sibutramine and its metabolites. Br J Pharmacol $1999 ; 126: 1487-95$.

9. Guerciolini R. Mode of action of orlistat. Int $\mathbf{J}$ Obes 1997;21(suppl.3):S12-S23.

10. Zhi J, Melia AT, Funk C, et al. Metabolic profile of mini mally absorbed orlistat in obese/overweight volunteers. J Clin Pharmacol 1996;36: 1006-11.

11. Bray GA, Blackburn GL, Ferguson JM, et al. Sibutramine produces dose-related weight loss. Obes Res $1999 ; 7: 189-98$.

12. Sjöstrom L, Rissanen A, Andersen T, et al. Randomized placebo-controlled trial of orlistat for weight loss and prevention of weight regain in obese patients. Lancet 1998:352:167-72.

13. Hollander PA, Elbein SC. Hirsch IB, et al. Role of orlistat in the treatment of obese patients with type 2 diabetes. Diabetes Care 1998:21:1288-94.

14. Davidson $\mathrm{MH}$, Hauptman J, DiGirolamo M, et al. Weight control and risk factor reduction in obese subjects treated for 2 years with orlistat. JAMA 1999;281:235-42.

\section{Endereço para correspondência:}

Alfredo Halpern

Rua Romilda Margarida Gabriel 81

04530-090 São Paulo, SP 